\title{
Visual search for global/local stimulus features in humans and baboons
}

\author{
CHRISTINE DERUELLE and JOËL FAGOT \\ Center for Research in Cognitive Neurosciences, Marseille, France
}

\begin{abstract}
Fagot and Deruelle (1997) demonstrated that, when tested with identical visual stimuli, baboons exhibit an advantage in processing local features, whereas humans show the "global precedence" effect initially reported by Navon (1977). In the present experiments, we investigated the cause of this species difference. Humans and baboons performed a visual search task in which the target differed from the distractors at either the global or the local level. Humans responded more quickly to global than to local targets, whereas baboons did the opposite (Experiment 1). Human response times (RTs) were independent of display size, for both local and global processing. Baboon RTs increased linearly with display size, more so for global than for local processing. The search slope for baboons disappeared for continuous targets (Experiment 2). That effect was not due to variations in stimulus luminance (Experiment 3). Finally, variations in stimulus density affected global search slopes in baboons but not in humans (Experiment 4). Overall, results suggest that perceptual grouping operations involved during the processing of hierarchical stimuli are attention demanding for baboons, but not for humans.
\end{abstract}

Navon (1977) suggested that the visual perception of hierarchical objects proceeds from a global analysis to processing of local details. The main argument supporting this hypothesis is that when hierarchical stimuli are processed, response times (RTs) are faster for global than for local targets (see, e.g., Kimchi, 1988), although the global advantage may disappear or even turn to a local advantage under certain circumstances (e.g., when there are only a few local elements; Martin, 1979).

Interestingly, although Navon (1991) suggested that the effect of global precedence is adaptive, only a handful of studies have investigated this effect in animals. Horel (1994) demonstrated that cooling the dorsal inferotemporal cortex of fascicularis monkeys inhibited their capacity to recognize local, but not global, stimulus properties. More recently, Hopkins (1997) demonstrated that chimpanzees exhibit a right visual field (left-hemisphere) advantage for the processing of local cues, and no significant asymmetry for the discrimination of global cues. Using a matching-to-sample task, Fagot and Deruelle (1997) demonstrated a strong advantage for local processing in baboons. That advantage remained when some physical aspects of the stimuli were manipulated, such as their shape or the connectedness of their local elements. In contrast, humans who were tested in the same experimental conditions as baboons showed the advantage for global processing reported by Navon (1977).

We thank B. Arnaud, G. Argenton, M. Chiambretto, R. Fayolle, and F. Lavergne for technical assistance. J. Requin and B. Sharf are acknowledged for their comments on an earlier version of this manuscript Correspondence should be addressed to $\mathrm{C}$. Deruelle, Center for Research in Cognitive Neurosciences, CNRS, 31 chemin Joseph-Aiguier, 13402 Marseille, Cedex 20, France (e-mail: chris@lnf.cnrs-mrs.fr).
The main objective of the present research was to assess the reliability of the local advantage in baboons and to understand species differences in the processing of hierarchical stimuli. To this end, we adopted a visual search procedure such as that used with humans by Saarinen (1994) and Enns and Kingstone (1995). In visual search tasks, subjects report whether or not a target stimulus is present in a display containing various distractors. A critical variable is the number of distractors in the display, and the experimenter measures RTs depending on display size. The rationale of this task (see, e.g., Treisman \& Gelade, 1980) is that baseline RTs reflect preattentive stages of processing, in which stimulus features are processed in parallel across the visual field. In turn, the involvement of attentional (serial) search operations is demonstrated when the slope of the relationship between RTs and display size is significant.

\section{EXPERIMENT 1}

In Experiment 1, a visual search task was adopted for which the target differed from the distractor at either the local or the global level. Humans and baboons were tested under identical experimental conditions and with the same stimuli, thus permitting a direct comparison of performance across species. Results of this experiment were published in an abbreviated form in Deruelle and Fagot (1997).

\footnotetext{
Method

Subjects. Monkeys were the same eight 7-year-old baboons (Papio papio; 4 males, 4 females) as in Fagot and Deruelle (1997). They were not food deprived, but received their food ration after completion of daily training or testing sessions. Eight human participants ( 4 men and 4 women, 22-28 years of age) were also tested. The humans were never informed of the purpose of the experiment and were paid for their participation
} 
Target

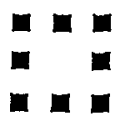

Experiment 2

Experiment 4

Large-sparse

Experiment 4

Small-dense

Experiment 4
Large-dense
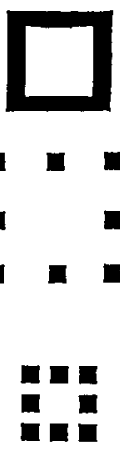

Global distractor
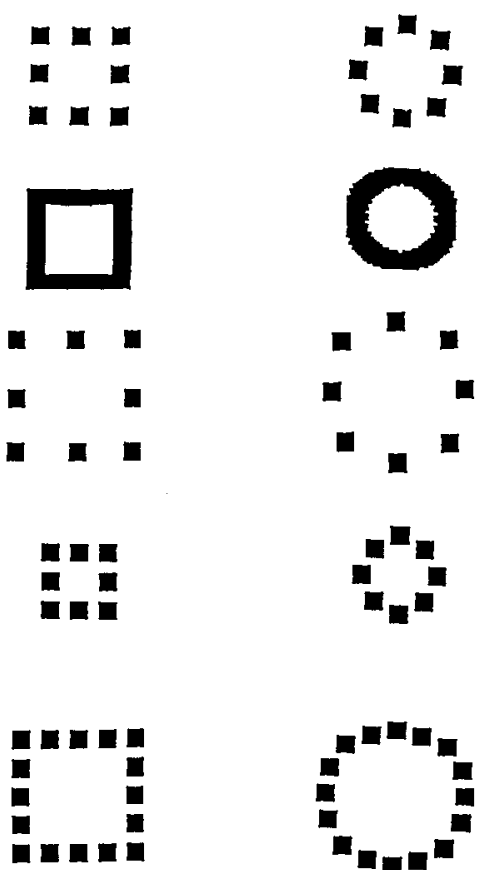

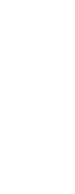
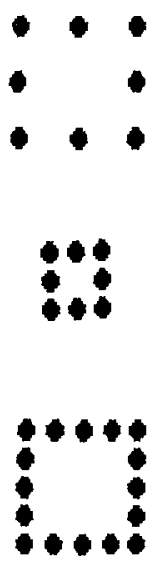

Figure 1. Stimuli used in Experiments 1, 2, and 4.

Apparatus. The apparatus consisted of a 14-in. color monitor driven by a 386 PC-AT computer, and an analog joystick. Baboons were tested in a cage $(68 \times 50 \times 72 \mathrm{~cm})$ facing the joystick and the monitor. The cage was fitted with a view port, two hand ports, and a food dispenser delivering food pellets within the cage. The testing apparatus for humans was the same as for baboons except that the monitor and joystick were laid on a table. Moreover, a chin bar, instead of a view port, was used to control the viewing distance to the monitor. For both humans and baboons, this distance was set at $49 \mathrm{~cm}$.

Stimuli. Figure 1 shows the stimulus set. Stimuli were large yellow squares or circles constructed from smaller squares or circles presented on a black background. The global shape of each stimulus subtended $3.0^{\circ} \times 3.0^{\circ}$ of visual angle and contained eight local elements of $.6^{\circ}$ of visual angle. Luminance of the stimuli was $99 \mathrm{~cd} / \mathrm{m}^{2}$, and the background was $5 \mathrm{~cd} / \mathrm{m}^{2}$.

Testing Procedure. For all subjects, a go/no-go procedure was used. Subjects initiated a trial by manipulating the joystick in order to move a cursor appearing in the center of the monitor screen within a $.5 \times$ $.5 \mathrm{~cm}$ square-shaped starting stimulus located $2 \mathrm{~cm}$ above or below the cursor. Once the cursor was correctly positioned for $35 \mathrm{msec}$ on the starting stimulus, a display comprising several compound stimuli appeared on the screen. For the no-go trials, all the forms of the display were large squares made up of small squares. For the go trials, all the stimuli were large squares constructed from small squares, except the target, which differed from the other forms (i.e., the distractors) at either the global or the local level. Accordingly, in local-go trials, the target was a large square made up of circles, whereas in global-go trials, the target was a large circle made up of small squares. The task was to move the joystick when the target was present (go trial) and to refrain from moving it when the target was absent (no-go trial). A no-go response was considered as correct when no joystick movement was detected by the computer during the $3 \mathrm{sec}$ following the onset of the display. A go re- sponse was considered correct when, within those $3 \mathrm{sec}$, subjects moved the joystick in any direction by an amplitude of at least 150 pixels.

Each subject served in four consecutive test sessions of 120 trials each. Within a session, 60 no-go trials were intermixed randomly with 30 local-go and 30 global-go trials. For each trial type, there was an equal number of trials per display size condition. Display sizes were set to 4,8 , or 12 items. Overall, 10 different displays were used per display size $X$ test condition (i.e., no-go, global-go, local-go). These displays were created according to the three following rules. First, $1^{\circ}$ of visual angle minimum separated the compound forms in the display. Second, the maximum possible display area was held constant (i.e., $640 \times$ 480 pixels) across set sizes. Third, alignments of the compound forms in either the vertical or the horizontal axes were prevented.

Pretraining for monkeys and humans. Baboons initially learned the go/no-go responses on the basis of stimuli different from those used during the actual test. They were also trained with the compound stimuli shown in Figure 1, but with one, three, or five stimuli per visual display. These three display sizes were mixed within a training session. Half of the monkeys were trained, first, to search for the local target and then to search for the global target. The other half were trained in the reverse order. Training continued until performance reached $80 \%$ correct in a block of 120 trials (i.e., 60 go trials intermixed with 60 no-go trials). Finally, the baboons were trained in blocks of 120 trials in which 30 local-go and 30 global-go trials were randomly intermixed with 60 no-go trials until they reached the $80 \%$ criterion in one block.

Prior to the test, humans were shown how to initiate the trials and to manipulate the joystick. The instruction emphasized the need to respond as fast as possible in the go trials. Subjects were allowed $10-30$ practice trials in which the local-go and global-go trials were intermixed with no-go trials.

Data analysis. RTs were measured from the onset of the search display to the manipulation of the joystick at the requested amplitude of 
150 pixels. RTs and scores in this and follow-up experiments were analyzed using parametric analyses of variance (ANOVAs). For reasons of conciseness, only the significant effects of the highest order will be reported. When necessary, post hoc analyses were done using Tukey's honestly significant difference method $(p<.05)$ tests.

\section{Results}

Median RTs were analyzed by way of a species (human, baboon) $\times$ test condition (global-go, local-go) $\times$ display size $(4,8,12)$ ANOVA. The two-way species $\times$ display size interaction was significant $[F(2,28)=4.81, p<.01]$. For humans, there were no significant RT differences among the display sizes of $4(443 \mathrm{msec}), 8(446 \mathrm{msec})$, and $12(449 \mathrm{msec})$ items. For baboons, RTs were slower for the 12 -item $(510 \mathrm{msec})$ than for the 8 -item $(485 \mathrm{msec})$ or the 4-item $(469 \mathrm{msec})$ display sizes. The species $\times$ test condition interaction was significant $[F(1,14)=21, p<$ $.001]$. On the average, baboons showed a significant advantage for local trials (global, $510 \mathrm{msec}$; local, $466 \mathrm{msec}$ ), and humans showed a significant advantage for global trials (global, $421 \mathrm{msec}$; local, $470 \mathrm{msec}$ ). Finally, there was a marginally significant species $\times$ display size $\times$ test condition interaction $[F(2,28)=3.26, p=.05]$, which is displayed in Figure 2. Because that interaction was significant, trend analyses were performed, independently for each species and test condition, to determine whether RTs increased linearly with display size. For baboons, linearity accounted for $99 \%$ of the variance observed in the global-go trials $(p<.05)$. However, linearity did not account for a significant proportion of the variance for the local-go trials $(92 \%)$ in baboons or for the global-go $(21 \%)$ or the local-go $(1 \%)$ trials in humans.

Accuracy data for humans were not analyzed because of a likely ceiling effect $(M=99.5 \%$, range $=98.8 \%-99.7 \%)$. For baboons, accuracy $(M=90.1 \%$, range $=81 \%-98.7 \%)$ was analyzed in a testing condition (global, local) $\times$ display size $(4,8,12)$ ANOVA. Results were consistent with those obtained for RTs and provided no evidence for a speed-accuracy tradeoff.

\section{Discussion}

Consistent with previous observations by Fagot and Deruelle (1997), Experiment 1 showed a local advantage for baboons and a global advantage for humans. The local advantage for baboons was already apparent in the number of trials that was necessary to reach learning criterion during the pretraining phase $(M$ local $=349$ trials; $M$ global $=$ 984 trials, $t$ test, $p<.01$ ), suggesting that species differences in processing are unrelated to the length of training or to practice effects. It is noticeable that human RTs did not increase linearly with display size, whatever the testing condition. In contrast, RTs increased with display size for baboons, particularly in the global-go condition. Therefore, our data suggest that processing at the global level was attention demanding for the baboons. Less attention was needed to process the stimulus at the local level, which was also the case in humans for both the global and the local levels.

The global structure of the stimuli of Experiment 1 needs to be perceived as a whole, an operation in which local elements are grouped into a single percept. It might be that, contrary to humans, baboons had difficulty "perceptually grouping" the local elements of the forms. In Experiment 2, we tested this hypothesis by using a visual search task with continuous stimuli for which there was no need for a perceptual grouping operation.

\section{EXPERIMENT 2}

\section{Method}

The baboons, apparatus, training procedure, and go/no-go procedure were the same as in Experiment 1. However, in this experiment, the distractors were large continuous squares and the target was a large continuous circle (see Figure 1). The baboons received four test sessions of 60 no-go trials intermixed with 60 go trials.

\section{Results and Discussion}

Accuracy data were not analyzed for statistical significance because of a likely ceiling effect $(96.65 \%$ correct on the average). Mean RTs were 424, 430, and $441 \mathrm{msec}$ for the 4-, 8-, and 12-item displays, respectively. Trend analyses on these data revealed no significant search slopes.

Of particular importance for our purpose was the comparison between these results and those of Experiment 1 . Thus, a display size $(4,8,12) \times$ experiment (Experiment 1 , Experiment 2) ANOVA was conducted to compare median RTs in Experiment 2 with median RTs in the localgo trials of Experiment 1. The main effect of display size was significant $[F(2,14)=17.7, p<.001]$, showing that RTs increased with display size ( $4,440 \mathrm{msec} ; 8,446 \mathrm{msec}$; $12,460 \mathrm{msec})$. The effect of experiment was also statistically significant $[F(1,7)=7.04, p<.05]$, showing faster RTs in Experiment $2(M=431 \mathrm{msec})$ than in the local-go trials of Experiment $1(M=466 \mathrm{msec})$. The display size $\times$ experiment interaction was not significant $[F(2,14)=$ $0.3, p>.1$, suggesting that identical processes were at work for target detection in the two experiments.

Another ANOVA compared RTs of Experiment 2 with those of the global-go trials of Experiment 1. The display size $\times$ experiment condition interaction was statistically significant $[F(2,14)=3.71, p=.05]$. The effect of display size on RTs was found to be larger in the global-go trials of Experiment 1 than in Experiment 2 (differences were of 62,73 , and $99 \mathrm{msec}$ for the 4-, 8-, and 12-stimulus displays, respectively). Taken together, the results suggest that baboons can discriminate with a high level of performance continuous shapes having the same size as the global size used in Experiment 1. Moreover, given the structural difference (either continuous or not) between the stimuli used in Experiments 1 and 2, the findings suggest that the search slope observed in Experiment 1 (global-go trials) depended on perceptual grouping operations.

It should be noted that there was a confounded factor in the comparison between Experiment 1 (global condition) and Experiment 2 because the stimuli of these two experiments differed in terms of both connectedness and number of constituent pixels. Differences in results can thus be explained by variations in overall luminance instead of variation in the need for grouping operations. In Experiment 3, we addressed these two possibilities by 


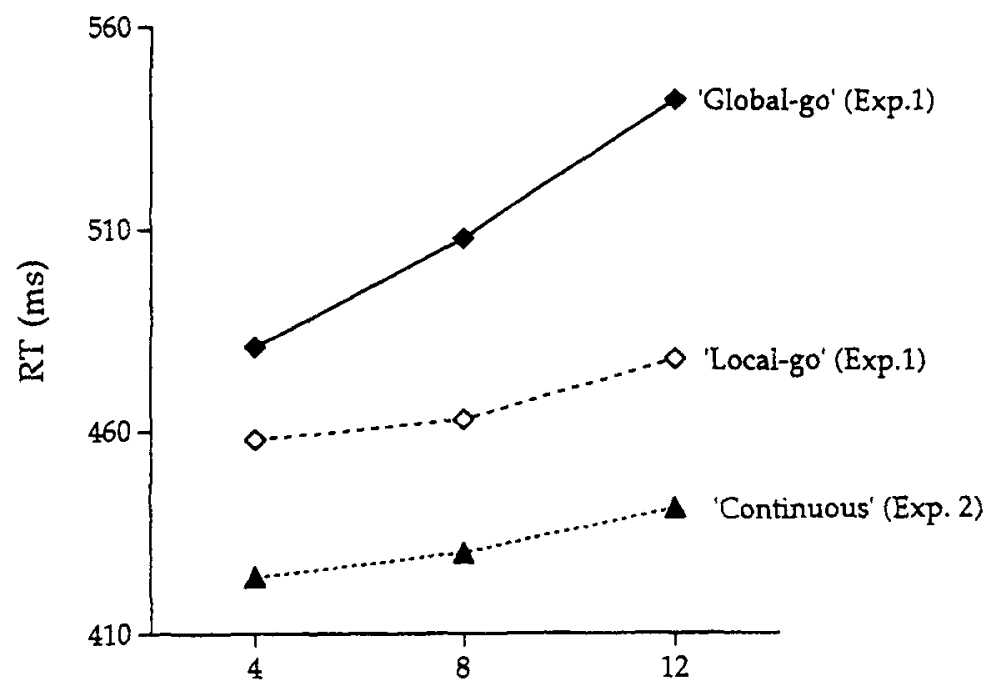

Display Size

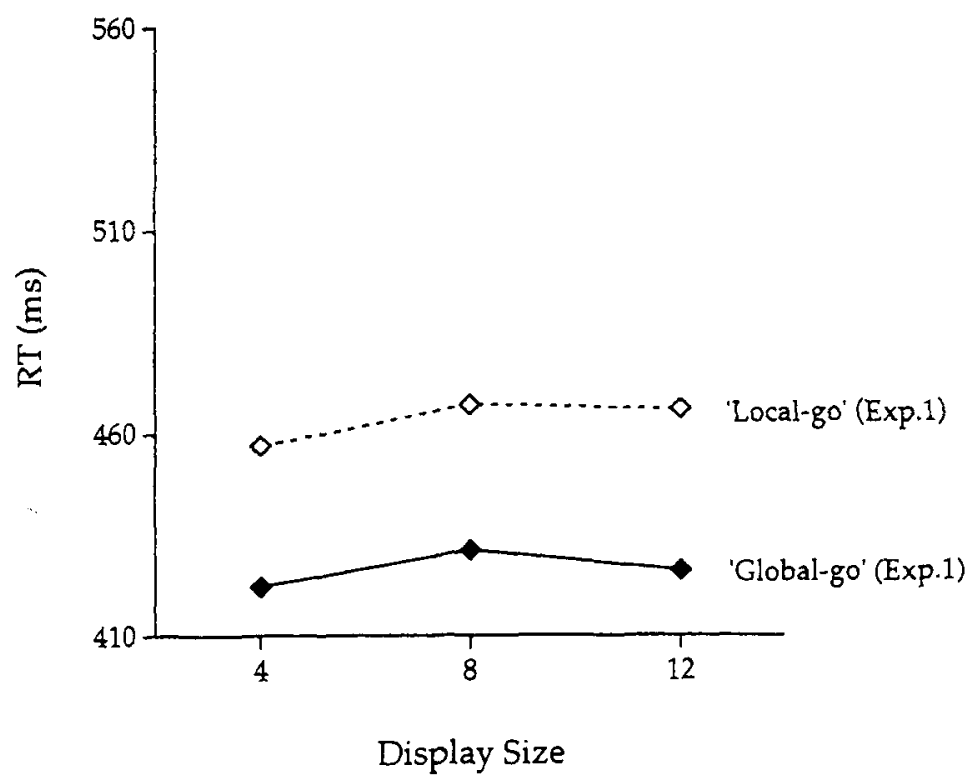

Figure 2. Baboons' (top) and humans' (bottom) response times (RTs) as a function of display size in the local-go and global-go trials of Experiment 1, and in Experiment 2.

manipulating the luminance factor and the need for perceptual grouping in an orthogonal way.

\section{EXPERIMENT 3}

\section{Method}

In Experiment 3, we used the discontinuous global trials of Experiment $l$ in combination with the continuous trials of Experiment 2. Moreover, the discontinuous and continuous stimuli had the same physical properties as in Experiments 1 and 2, respectively, but were either gray (low-luminance condition: $14 \mathrm{~cd} / \mathrm{m}^{2}$ ) or white (high-luminance condition: $110 \mathrm{~cd} / \mathrm{m}^{2}$ ).

Each baboon received two test sessions of 216 test trials each, one with the continuous stimuli and one with the discontinuous stimuli. The order of the two sessions was counterbalanced across individuals. Within each session, there were 18 trials per display size $(4,8,12) \times$ trial type (go, nogo) $\times$ luminance (low, high) condition. The order of the trials was randomly determined prior to the test and thus differed between subjects. Baboons received training prior to each testing session. The training procedure was identical to training in Experiments 1 and 2, but involved the gray and white stimuli, which were presented in blocked sessions. 


\section{Results}

Median RTs for correct responses were subjected to a stimulus type (continuous, discontinuous) $\times$ luminance (low, high) $\times$ display size $(4,8,12)$ ANOVA. The display size $\times$ stimulus type interaction reached statistical significance $[F(2,14)=10, p<.002]$. RTs increased linearly with display size ( $M 4=430 \mathrm{msec} ; M 8=484 \mathrm{msec}$; $M 12=555 \mathrm{msec}$ ) when discontinuous stimuli were used (linearity accounted for $99 \%$ of the variance, $p<.05$ ). By contrast, there was no significant search slope when continuous stimuli were used $(M 4=393 \mathrm{msec} ; M 8=$ $414 \mathrm{msec} ; M 12=413 \mathrm{msec}$ ). The luminance factor had no significant effect and did not interact significantly with any of the other experimental factors.

Accuracy was high on the average $(M=91.6 \%$ correct, $S D=11$ ). The ANOVA on scores replicated the results on RTs and provided no evidence for a speed-accuracy tradeoff. In brief, Experiment 3 failed to demonstrate a significant effect of luminance on search slope.

\section{EXPERIMENT 4}

Experiments 1-2 suggested that the mechanisms involved in perceptual grouping operations are at the origin of the previously observed human-baboon differences in global/local processing. In Experiment 4, we further addressed this hypothesis by changing the distance between local elements in order to manipulate the need for perceptual grouping.

\section{Method}

The same 8 baboons as before, and 8 humans ( 4 men and 4 women, 22-30 years of age) participated in this experiment. The stimuli were of a similar type as before, but were of different sizes and densities (see Figure 1). Stimulus density was manipulated either by changing the overall size of the global form (i.e., $2^{\circ}$ or $4^{\circ}$ of visual angle), by keeping the number of elements constant $(n=8)$, or by varying the number (i.e., 8 or 16 ) of local elements at constant stimulus size (i.e., $4^{\circ}$ ). There were therefore three different stimulus-type conditions: large sparse, large dense, and small dense. In order to display all the large stimuli on the screen, display sizes were set to 3,6 , or 9 items.

Each subject received four consecutive test sessions of 96 trials per stimulus-type condition, in which the global/local trials were intermixed. The order of test condition presentation was counterbalanced across individuals, although a completely balanced design was impossible given the even number of subjects. The other experimental details of the procedure were identical to those of Experiments 1-3.

\section{Results and Discussion}

Humans achieved a very high level of performance on average ( $>99 \%$ correct on average) that precluded an analysis of accuracy in which species was used as a factor. Baboons performed $81 \%$ correct on the average. Their performance was analyzed using a stimulus type (largesparse, large-dense, small-dense) $\times$ test condition (globalgo, local-go $) \times$ display size $(3,6,9)$ ANOVA.

The main effect of stimulus type was significant $[F(2,14)=38, p<.001]$, showing lower performance on the average in the large-sparse condition $(M=58 \%$ correct) than in the large-dense ( $M=94.5 \%$ correct) or the small-dense conditions ( $M=91.2 \%$ correct). There was also a significant stimulus type $\times$ test condition interaction $[F(2,14)=37.6, p<.001]$. Post hoc analyses revealed a significant local advantage in the large-sparse condition, but no significant difference between the global and the local trials of the large-dense and small-dense conditions. Moreover, the stimulus type $\times$ display size interaction was significant $[F(2,14)=12.7, p<.002]$. That interaction showed no significant effect of display size for either the large-dense or the small-dense conditions, but did show a linear increment in the number of error trials with display size for the large-sparse condition (linearity accounted for more than $99 \%$ of the variance, $p<.05$ ). Finally, the stimulus type $\times$ test condition $\times$ display size interaction was also significant $[F(4,28)=10.4, p<.001]$, showing, for the global trials of the large-sparse condition only, that the largest display sizes gave rise to the largest number of errors.

Baboon RTs were not analyzed because of the low accuracy in the large-sparse condition. For humans, median RTs were analyzed using a stimulus type $\times$ testing condition $X$ display size ANOVA. The significant main effect of stimulus type $[F(2,14)=12.6, p<.002]$ showed longer RTs on the average for the large-sparse $(M=432 \mathrm{msec})$ than for the large-dense condition $(M=387 \mathrm{msec})$. Mean RTs for the large-sparse condition did not differ significantly from RTs for the small-dense condition. The main effect of test condition was significant $[F(1,7)=27, p<$ $.002]$, showing faster RTs for the global $(M=397 \mathrm{msec})$ than for the local condition $(M=427 \mathrm{msec})$. There was no other significant main effect or interaction.

In brief, Experiment 4 confirmed that humans and baboons differ in their ability to perceive the global structure of Navon-type stimuli by way of perceptual grouping. In humans, the global advantage was replicated whatever the stimulus density condition, and no search slope was observed. In baboons, a local advantage and a performance decrement with display size were observed only for the global trials of the large-sparse condition. In the other stimulus type conditions, there were no significant advantages for either global or local trials, and no significant search slopes.

\section{GENERAL DISCUSSION}

Fagot and Deruelle (1997) demonstrated a local advantage in baboons for the processing of hierarchical stimuli. The present series of experiments confirmed that advantage and, moreover, provided clues for understanding it. Indeed, Experiment 1 showed that RTs increased with display size-more so for global than for local targets-thus demonstrating that the processing of the global stimulus level is more attention demanding than the processing of the local stimulus level. Complementarily, Experiments 2-4 showed that the search slope observed with the global targets depends predominantly on the attentional nature of the perceptual grouping mechanisms. It is proposed on the basis of these results that the local advantage of baboons reflects the direct access to the local features compared with access to the global ones, for which perception involves an additional (attentional) grouping operation.

Considering human subjects, the results of Experiment 1 confirmed the global precedence hypothesis proposed by Navon (1977). Interestingly, no evidence was found for a systematic relation between RTs and the number of stimuli contained in the display, whatever the stimulus 
level to be processed. This result contrasts with the previous findings by Enns and Kingstone (1995), who reported steeper search slopes in the global-go than in local-go trials. In agreement with the present data, however, Saarinen (1994) failed to report significant search slopes in the processing of the global and local aspects of compound-oriented lines. Further experiments should indicate whether a search slope would occur in humans when they are tested in our experimental conditions with the same stimuli as those in Enns and Kingstone.

One question that remains is whether or not physiological explanations can account for the human-baboon species differences in global/ local processing. Unfortunately, the literature provides very few psychophysical measurements on the functional sensibility of the visual system in baboons. Available data, however, show that the visual system of Old World primate species (which include the baboon species) share numerous properties with that of humans-for instance, when the distribution of the ganglion cells in the retina (Fischer \& Kirby, 1991) or the contrast sensitivity functions (De Valois \& De Valois, 1990) are considered. Although it is not possible to completely rule out the existence of subtle sensitivity perceptual differences between humans and baboons, these consistencies across primate species support the hypothesis that the differences we observed between baboons and humans have postperceptual (i.e., attentional) instead of purely perceptual origins.

\section{REFERENCES}

Deruelle, C., \& FAGot, J. (1997). Hemispheric lateralization and global precedence effects in the processing of visual stimuli by humans and baboons. Laterality, 2, 233-246.

DE Valors, R. L., \& DE ValoIs, K. K. (1990). Spatial vision. New York: Oxford University Press.

EnNS, J. T., \& Kingstone, A. (1995). Access to global and local properties in visual search for compound stimuli. Psychological Science, 6, 283-291.
Fagot, J., \& Deruelle, C. (1997). Processing of global and local visual information and hemispheric specialization in humans (Homo sapiens) and baboons (Papio papio). Journal of Experimental Psychology: Human Perception \& Performance, 23, 429-442.

FisCHER, Q. S., \& KIRBY, M. A. (1991). Number and distribution of retinal ganglion cells in anubis baboons. Brain Behaviour \& Evolution, 37, $189-203$

HopKINs, W. D. (1997). Hemispheric specialization for local and global processing of hierarchical visual stimuli in chimpanzees (Pan troglodytes). Neuropsychologia, 35, 343-348.

Horel, J. A. (1994). Local and global perception examined by reversible suppression of temporal cortex with cold. Behavioural Brain Research, 65, 157-164.

Kiмchi, R. (1988). Selective attention to global and local levels in the comparison of hierarchical patterns. Perception \& Psychophysics, 43, 189-198.

MARTIN, M. (1979). Local and global processing: The role of sparsity. Memory \& Cognition, 7, 476-484.

NAVON, D. (1977). Forest before the tree: The precedence of global features in visual perception. Cognitive Psychology, 9, 353-383.

Navon, D. (1991). Testing a queue hypothesis for the processing of global and local information. Journal of Experimental Psychology: General, 120, 173-189.

SAARINEN, J. (1994). Visual search for global and local stimulus features. Perception, 23, 237-243.

Treisman, A., \& Gelade, G. (1980). A feature integration theory of attention. Cognitive Psychology, 12, 97-136.

(Manuscript received June 3, 1997; revision accepted for publication November $24,1997$. 